\title{
SOLITARY FIBROUS TUMOR OF THE PLEURA: \\ A SIMPLE SOLUTION WITH A SIMPLE TECHNIQUE
}

Muhammad Hafiz Ismail, Loo Guo Hou, Hairolfaizi Haron, Mohd Ramzisham bin Abdul Rahman

Department of surgery, Faculty of Medicine Universiti Kenbangsaan Malaysia Medical Center, Jalan Yqacob Latiff, Bandar Tun Razak, 56000, Kuala Lumpur, Malaysia.

\section{ABSTRACT}

Surgery has been the mainstay of treatment in managing patients with lung or pleural tumor. Surgery can be in the form of video assisted or open technique. Preoperative investigation such as Computed Tomography (CT) scan of the thorax has been an important tool of assessment in deciding the approach of surgery. Traditionally, a tumor of more than $6 \mathrm{~cm}$ would necessitate an open approach. Herein, we would like to report a patient with a large solitary fibrous tumor of the pleura which was thoracoscopically excised and evacuated in an unconventional approach.

\section{Keywords:}

\section{Corresponding Author:}

Dr. Muhammad Hafiz Ismail - MBBS, MRCS (Edinburgh)

Address: Department of surgery, Faculty of Medicine Universiti Kenbangsaan Malaysia Medical Center, Jalan Yqacob Latiff, Bandar Tun Razak, 56000, Kuala Lumpur, Malaysia.

E-mail: muhdhafizismail88@gmail.com

Copyright $(\mathcal{C}$ 2012- 2021 Muhammad Hafiz Ismail, et al. This is an open access article published under Creative Commons Attribution -Non Commercial- No Derives 4.0 International Public License (CC BY-NC-ND). This license allows others to download the articles and share them with others as long as they credit you, but they can't change them in any way or use them commercially.

doi: 10.46327/msrig.1.000000000000----

\section{CASE REPORT}

A 57-year-old man with a background of chronic active smoker was referred for an incidental finding of right hilar mass on chest radiograph for elective hernia surgery. A standard Chest CT performed revealed the presence of a lobulated, non-enhancing soft tissue lesion at the right anterior mediastinal region measuring $5.0 \times 6.4 \mathrm{~cm} \times 7.8 \mathrm{~cm}$ in diameter with no clear fat plane anterior to this mass (figure 1 and 2).

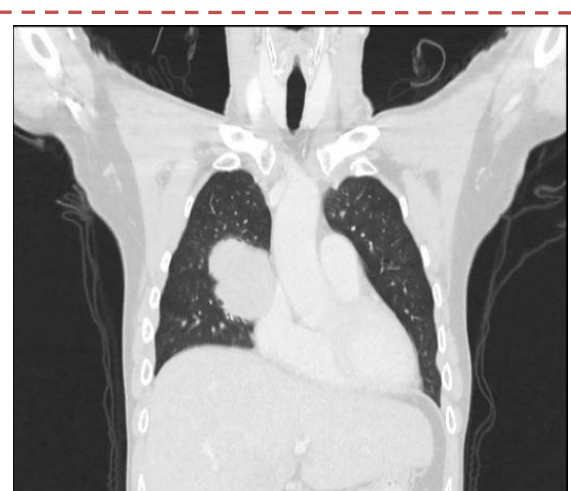

Figure 1: coronal view of CT thorax showing a lobulated lesion at the right hilum. 


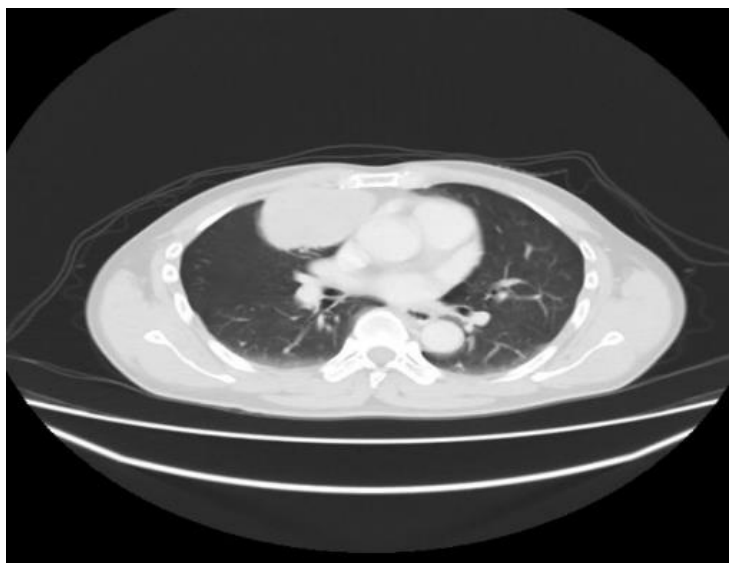

Figure 2: axial view of a CT thorax demonstrates loss of fat plane between the lesion and soft tissues anteriorly

Thereafter, a whole-body Fluorodeoxyglucose Positron Emission Tomography (FDG PET) -CT scan performed showing the lesion demonstrates a mild FDG metabolism with no hypermetabolic lesions elsewhere (Figure 3). A percutaneous needle biopsy of the lesion was done before commencing on surgery demonstrates low grade spindle cells.

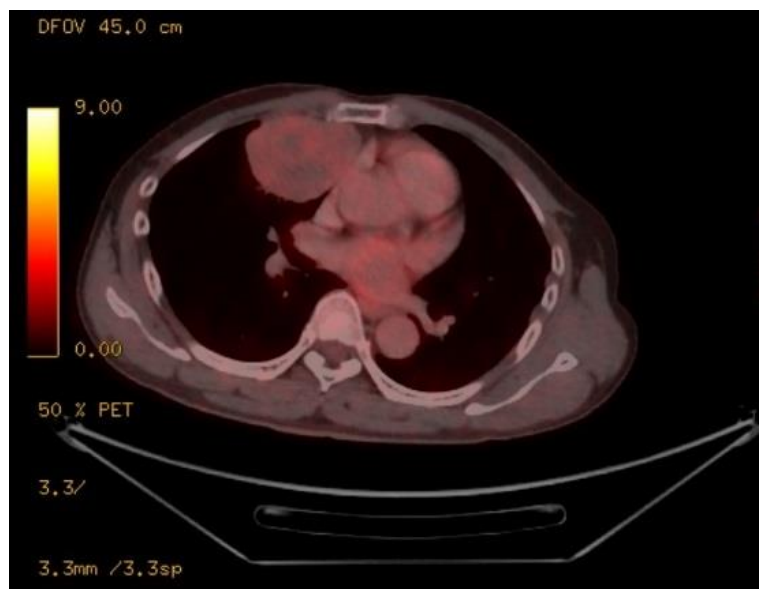

Figure 3: An FDG PET-CT scan demonstrating a lesion with mild FDG metabolism

A Uniportal Video Assisted Thoracoscopic Surgery (VATS) was performed revealing an $8 \mathrm{~cm}$ in size pedunculated tumor arising from the visceral pleura of the right middle lobe. In contrast to the CT thorax findings, the tumor was freely mobile and did not infiltrates the surrounding tissue. Macrospically, the nodule appears yellowish, firm, and well demarcated suggesting a benign tumor (Figure 4).

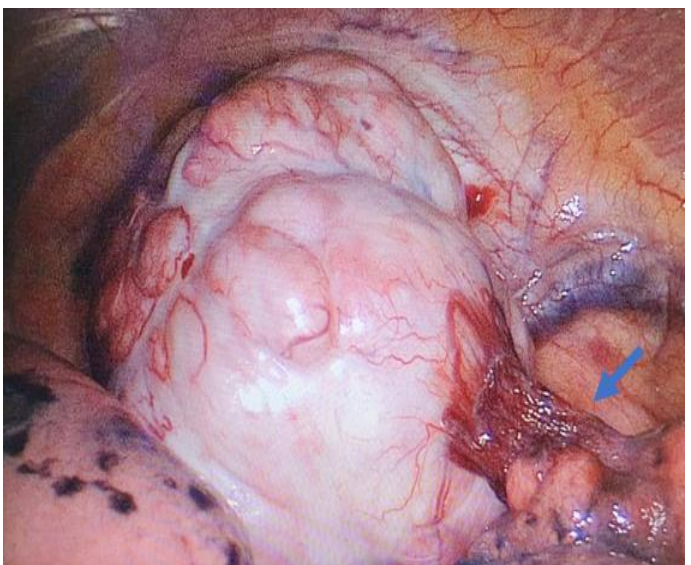

Figure 4: A lobulated, well circumscribed lesion with peduncle attached to the visceral pleura of middle lobe (blue arrow)

Wedge resection was performed using linear stapler at its peduncle base. The lesion was divided into several small pieces inside a pouch bag prior to evacuation from the thoracic cavity (Figure 5).

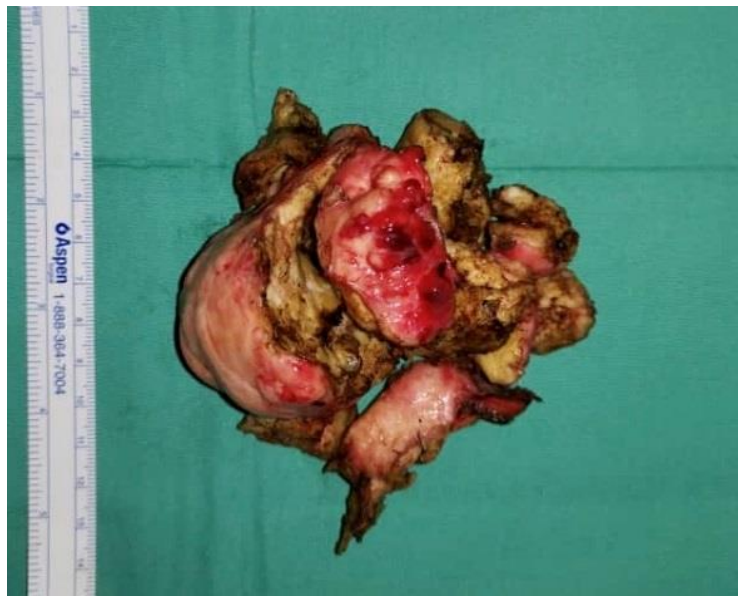

Figure 5: Lesion was fragmented to help in evacuation from the thoracic cavity via a small port incision

The histopathological findings showed a wellcircumscribed tumour composed of uniform fibroblastic spindle cells with presence of prominent stromal hyalinisation and collagen. Immunohistochemically, the tumor cells show strong and diffuse reactivity for CD34 and bcl-2. Focal and weak expression of beta-catenin and Smooth Muscle Actin (SMA) are present while stains for S100, Epithelial Membrane Antigen (EMA) and Desmin are negative.

His postoperative course was uncomplicated. There has been no recurrence in the 12 months postsurgery. 


\section{DISCUSSION}

Solitary Fibrous Tumor of the Pleura (SFT) is a rare tumor that accounts for $5 \%$ of all pleural tumor. It is commonly presented in the 5th and 6th decade of life and equal in both men and women [1]. Most are asymptomatic and found incidentally due to other reason such as preoperative assessment for other surgery. SFT usually presented as a pedunculated well circumscribed mass. More often, the peduncle has tendency to be attached to visceral pleura rather than parietal pleura [2]. Symptoms occurred mostly due to compressive symptoms such lung collapse, atelectasis or even inferior vena cava obstruction The gold standard of diagnosing and assessment of SFT is CT scan of the chest. It allows a good visualization of the parietal, visceral and mediastinal pleura and provides the required information regarding adjacent lung collapsed, bony involvement and presence of pleural effusion. Although chest CT remains the gold standard diagnostic imaging, differentiating between malignant and benign pleural tumor remains relatively dubious. According to Ferretti et al., however, the important criteria in suspecting a malignancy are: (1) central necrosis, (2) tumor diameter $>10 \mathrm{~cm}$ and (3) ipsilateral pleural effusion [3]. In our patient, none of the mentioned criteria were present in his chest CT, however, the pleural mass appeared to be invading the anterior chest wall, thus increased the suspicion of possible malignancy. Therefore, a whole body FDG-PET CT scan was performed to exclude possible malignancy. It is important to note that any mesenchymal tumor, either benign or malignant may mimic each other radiologically. Hence, the final diagnosis remains pathological [4].

SFTs can be diagnosed by percutaneous transthoracic needle biopsy, although it provides a low yield positive fine-needle aspiration (FNA), which is $43 \%$ [5]. Hence, it is commonly misdiagnosed as other diseases, such as thymic neoplasia, teratoma, neurogenic tumor, malignant pleural mesothelioma or lung cancer.

The mainstay of treatment of SFT is surgery. Following a complete surgical resection, the prognosis of pleural SFTs can be excellent, while careful follow-up is still necessary [6]. Surgery can be in the form of thoracotomy or video assisted, although the latter is a much more preferred technique in the recent years.

In our case, preoperative investigation showed the tumor may be malignant as it demonstrates local infiltration features and it is relatively big in size. Therefore, dilemma arose pertaining to the best approach for surgery; either VATS or thoracotomy. While VATS may appear as a relative contraindication due to large lesion size that may limit visibility (a threshold of $6 \mathrm{~cm}$ has been previously reported) [7], a thoracotomy would lead to unnecessary large incision and poor postoperative recovery. Hence, we opted for VATS for intraoperative assessment first. As in our case, the tumor was pedunculated with a biggest diameter of $9 \mathrm{~cm}$ and it was successfully excised at its base. To prevent transfer of tumor cells and metastases, the tumor was divided into small pieces in the endoscopic pouch.

\section{CONCLUSION}

VATS should be the primary approach for surgical resection of lung tumor whenever possible. It provides a commendable intraoperative assessment and superior perioperative outcome.

\section{REFERENCES}

1. Ichiki, Y. et al. Solitary fibrous tumor of the lung: a case report. Surgical Case Reports 3, 10 (2017).

2. Ordóñez, N. G. Localized (Solitary) Fibrous Tumor of the Pleura. Advances in Anatomic Pathology 7, 327-340 (2000).

3. Ferretti, G. R., Chiles, C., Choplin, R. H. \& Coulomb, M. Localized benign fibrous tumors of the pleura. American Journal of Roentgenology 169, 683-686 (1997).

4. Hammas N, Douida A, Serghouchni K I, Chbani L, El Fatemi H. Malignant solitary fibrous tumor: a report of two cases. Journal of medical and Surgical Research -JMSR 2018; V (1): 508511.

5. Sung, S. H. et al. Solitary Fibrous Tumors of the Pleura: Surgical Outcome and Clinical Course. The Annals of Thoracic Surgery 79, 303-307 (2005).

6. Lu C, Ji Y \& Shan F. solitary fibrous tumor of the pleura: an analysis of 13 cases. World J Surg 32, 8 (2008).

7. Agzarian, J. \& Shargall, Y. Open thoracic surgery: video-assisted thoracoscopic surgery (VATS) conversion to thoracotomy. Shanghai Chest 1, 31-31 (2017). 